\title{
MODELLING OF THE EFFECT OF THE FOREGOING WAKE ON THE BYPASS TRANSITION ON THE AIRFOIL
}

\begin{abstract}
Petr STRAKA, Jaromír PŘíHODA, David ŠIMURDA
Abstract: A modified algebraic model of the bypass transition was used for the simulation of the flow around the symmetrical airfoil NACA 0012 in the tandem configuration. The transition model is based on local variables only to enable its application for complex flow geometry using unstructured computational grids. The attention was especially focused on the flow near the leading edge where the boundary layer is not yet fully developed while used empirical correlations were established for the boundary-layer flow. The numerical simulation was compared with experiments of Lee and Kang [1] carried out for the Reynolds number $R_{c}=$ $(2 \div 6) \times 10^{5}$, the zero angle of attack and various distance between both airfoils. The agreement of numerical simulation with experimental data is quite satisfactory
\end{abstract}

\section{INTRODUCTION}

An adequate transition model must be used for the appropriate modelling of flow over airfoils in the aeronautics and through blade cascades in the turbomachinery. While in the external aeronautics simple transition models, as e.g. the $\mathrm{e}^{\mathrm{N}}$ model (Stock, Haase [2]), can be used because of very low free-stream turbulence level, the bypass transition model used for the prediction of internal flows with high free-stream turbulence level must be more sophisticated. Most of bypass transition models are mostly based on the concept of the intermittency coefficient according to Narasimha [3] and/or of the laminar kinetic energy (see Walters, Leylek [4]). Besides transition models with a transport equation for the intermittency coefficient, as Suzen, Huang [5], Langtry [6] or Langtry, Menter [7], Lodefier et al. [8], there are models with the algebraic relation for the intermittency coefficient, e.g. Thermann, Niehuis [9] and/or Straka, Příhoda [10]. Nevertheless both types of transition models need empirical relations describing the onset and the length of the transition region.

The adequate modelling of the laminar/turbulent transition forms an import part of the prediction of shear flow over airfoils and/or turbine blades especially in the case for flow with heat transfer. As well the appropriate modelling of flow near the leading edge is crucial. The presented bypass-transition model is based on the algebraic equation for the intermittency coefficient and empirical correlations for the Reynolds number characterizing the transition onset and for the parameter describing the origin and growth of turbulent spots depending on the pressure gradient and free-stream turbulence. The momentum Reynolds number is replaced by the vorticity Reynolds number for the

\footnotetext{
- Ing. Petr Straka, PhD., Aeronautical Research and Test Institute, Plc, Beranových 130, 19905 Praha - Letňany, straka@vzlu.cz

Prof. Ing. Jaromír Př́hoda, CSc., Institute of Thermomechanics AS CR, v.v.i., Dolejškova 5, 18200 Praha 8, prihoda@it.cas.cz

Ing. David Šimurda, PhD., Institute of Thermomechanics AS CR, v.v.i., Dolejškova 5, 18200 Praha 8, simurda@it.cas.cz
} 
application in complex geometries. The model distinguishes the different intermittency coefficient in the outer flow and in the boundary layer and so it gives the appropriate free-stream turbulence in internal flows with increasing turbulence level. The model was tested using standard test cases given the ERCOFTAC database and flow through the turbine blade cascade (see Straka and Př́íhoda [10]).

\section{MAthematical MOdel}

The mathematical model of compressible turbulent flow is based on the Favre-averaged Navier-Stokes equations. The system of governing equations is closed by the twoequation $k-\omega$ turbulence model proposed by Kok [11]

$$
\begin{gathered}
\frac{\partial(\rho k)}{\partial t}+\frac{\partial\left(\rho u_{j} k\right)}{\partial x_{j}}=\tilde{P}_{k}-\tilde{D}_{k}+\frac{\partial}{\partial x_{j}}\left[\left(\mu+\sigma_{k} \mu_{t}\right) \frac{\partial k}{\partial x_{j}}\right] \\
\frac{\partial(\rho \omega)}{\partial t}+\frac{\partial\left(\rho u_{j} \omega\right)}{\partial x_{j}}=\alpha_{\omega} \frac{\omega}{k} P_{k}-\beta_{\omega} \rho \omega^{2}+\frac{\partial}{\partial x_{j}}\left[\left(\mu+\sigma_{\omega} \mu_{t}\right) \frac{\partial \omega}{\partial x_{j}}\right]+\sigma_{d} \frac{\rho}{\omega} \max \left(\frac{\partial k}{\partial x_{j}} \frac{\partial \omega}{\partial x_{j}}, 0\right)
\end{gathered}
$$

where $\tilde{P}_{k}$ and $\tilde{D}_{k}$ are the production and destruction terms in the $k$ - equation modified in the laminar and transitional part of the shear layer, see Straka and Príhoda [10]). The turbulent viscosity is given by the relation

$$
\mu_{\text {to }}=\max \left(\frac{\rho k}{\omega} ; \sigma \sqrt{\frac{\mu}{\beta^{*} \omega k}}\right)
$$

where the Kolmogorov time scale is used for the description of the turbulent motion in the vicinity of the wall according to Durbin [12]. For the reduction of the energy production near the leading edge, the turbulent viscosity is given by the relation

$$
\mu_{t}=\mu_{t o} \min \left(1 ; \frac{\alpha}{\sqrt{3}} \frac{\omega}{\sqrt{2 S_{i j} S_{i j}}}\right)
$$

with constant $\alpha=0.6$. This restriction proposed by Medic and Durbin [13] is applied to the production of turbulent energy only. The effective viscosity is modified by the intermittency coefficient according to the relation

$$
\mu_{\mathrm{ef}}=\mu+\gamma \mu_{\mathrm{t}}
$$

The governing equations are discretized by means of the finite volume method using a multi-block quadrilateral structured grid with a block overlapping implementation. The finite volume method of the cell-centered type with the Osher's-Solomon's approximation of the Riemann solver and a two-dimensional linear reconstruction with the Van Albada's limiter was developed. The viscous fluxes are discretized in the central manner on a mesh dual to the cell faces. Time integration is performed with the linearized Euler backward formula in the implicit formulation. Equations for turbulent scales $\mathrm{k}$ and $\omega$ are solved separately from governing equations. After linearization, the system of linear equations is solved with the GMRES method with the ILU precondition.

\section{TRANSITION MODEL}

The algebraic bypass-transition model is based on the concept of different values of the intermittency coefficient in the boundary layer $\left(\gamma_{i}\right)$ and in the free stream $\left(\gamma_{e}\right)$. The smooth link-up between both zones is considered by the relation

$$
\gamma=\frac{\gamma_{i}+\gamma_{e}}{2}+\frac{\gamma_{e}-\gamma_{i}}{2} \operatorname{tgh}\left[C_{\gamma}\left(\frac{y}{\delta_{995}}-1\right)\right]
$$


with the constant $C_{\gamma} \approx 12 \div 18$.

The algebraic model of the bypass transition is based on the empirical relation for the inner intermittency coefficient

$$
\gamma_{i}=1-\exp \left[-\hat{n} \sigma\left(R e_{x}-R e_{x t}\right)^{2}\right]
$$

according to Narasimha [3]. The transition onset is given by the Reynolds number $R e_{x t}$ determined by means of the momentum Reynolds number $R e_{\vartheta t}$ depending on the freestream turbulence level $T u(\%)$ and the pressure-gradient parameter $\lambda_{t}$. The transition onset is given by the empirical correlation (see Straka and Príhoda [10])

$$
R e_{\vartheta t}=R e_{\vartheta t o}\left[1+\mathrm{F}(T u) \frac{1-\exp \left(-40 \lambda_{t}\right)}{1+0.4 \exp \left(-40 \lambda_{t}\right)}\right]
$$

where the transition onset on the flat plate without pressure gradient is given by the relation

$$
\begin{array}{rr}
R e_{\text {gto }}=975.8-497.2 T u+\frac{11.4}{T u} & \text { for } \quad T u \leq 1 \% \\
R e_{\text {gto }}=96.7+\frac{340}{T u}+\frac{53.3}{T u^{2}} & \text { for } T u>1 \%
\end{array}
$$

The function $\mathrm{F}(T u)$ is given by the relation

$$
F(T u)=0.29[1-0.54 \exp (-3.5 T u)] \exp (-T u)
$$

The correlation can be used for the free-stream turbulence in the range $0.1 \% \leq T u \leq 8 \%$ and the pressure-gradient parameter in the range $-0.1 \leq \lambda_{t} \leq 0.1$. The length of the transition region is given by parameters describing spot generation rate $n$ and spot propagation rate $\sigma$. Narasimha [14] proposed the correlation

$$
N=\hat{n} \sigma R e^{3}
$$

The effect of the free-stream turbulence and the pressure gradient is correlated by an empirical relation $N=\mathrm{f}\left(T u, \lambda_{t}\right)$ proposed by Solomon et al. [15]. According to Langtry and Menter [7], the maximum of the vorticity Reynolds number

$$
R e_{v \max }=\max \left(y^{2}|\Omega| / v\right)
$$

is used to avoid the calculation of the momentum Reynolds number $R e_{\vartheta}$ in cases with complex flow geometry. This link is given by the relation

$$
R e_{\vartheta}=\frac{R e_{v \max }}{C}
$$

where $C=2.185$ for the Blasius laminar boundary layer. Using similar solutions of Falkner and Skan [16], the parameter $C$ was expressed as a function of the modified pressure-gradient parameter

$$
L=R e_{v \max }^{2} \frac{v}{U_{e}^{2}} \frac{d U_{e}}{d x}
$$

The boundary layer thickness $\delta_{995}$ is similarly given by the relation $\delta_{995} / y_{\max }=g(L)$ where $y_{\max }$ is the position of the maximum vorticity Reynolds number.

Relevant values of free-stream turbulence are taken at the outer boundary of the shear layer estimated by means of the position of the maximum vorticity Reynolds number. Near the leading edge, especially in the wake of the upstream located airfoil, it is not easy to establish relevant values. On the other hand, the characteristic Reynolds numbers are in the accelerated flow permanently lower than the critical Reynolds number. Therefore, the criterion for the transition onset given by Eq. (8) was applied 
starting from the pressure minimum position, i.e. approx. from $x / c \approx 0.1$ for the NACA 0012 airfoil.

\section{Results}

The numerical simulation of the bypass transition was focused on the modelling of flow over the NACA 0012 airfoil in a tandem configuration according the experiment of Kang and Lee [1]. Experiments were accomplished in a closed-type wind tunnel where two NACA 0012 airfoils were mounted in the test section $600 \times 2000 \mathrm{~mm}$. The second airfoil is installed in the wake generated by the foregoing airfoil aligned in tandem. Measurements were carried out for the Reynolds number $R e_{c}$ in the range from $2 \times 10^{5}$ to $6 \times 10^{5}$, freestream turbulence level $T u=0.3 \%$, the zero angle of attack and various distance between both airfoils varies from $t / c=0.25$ to 1.0 of the chord length. The computational domain corresponds to the experimental arrangement. The detail of the computational mesh around the NACA 0012 airfoil with a quadrilateral structured grid near the surface is shown in Fig.1.

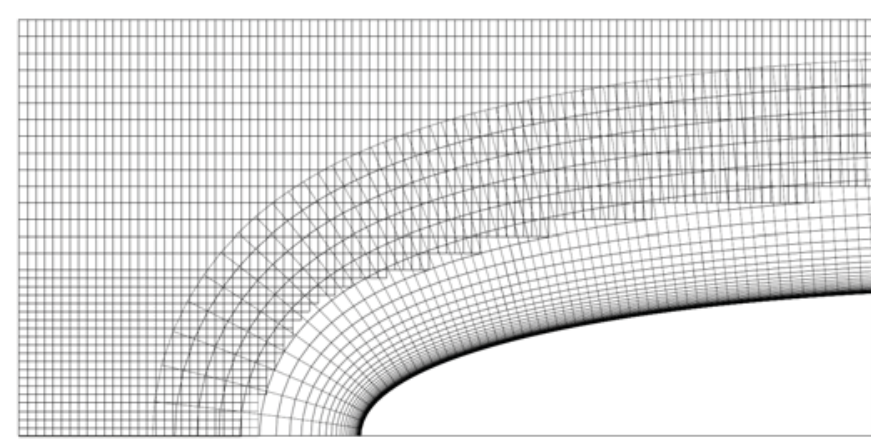

Figure 1: Detail of the computational mesh

As the basic configuration, flow over one airfoil at the Reynolds number $R e_{c}$ $=6 \times 10^{5}$ was studied (CASE 0 ). The effect of the Reynolds number was investigated for the distance $t / c=1$ and $R e_{c}=2 \times 10^{5}$ (CASE 3), $4 \times 10^{5}$ (CASE 2) and $6 \times 10^{5}$ (CASE 1). The effect of the distance between both airfoils was studied for the Reynolds number $\operatorname{Re}_{c}=$ $2 \times 10^{5}$ and the distance $t / c=0.5$ (CASE 4) and 0.25 (CASE 5).

The distribution of the pressure coefficient $C_{p}$ and the friction coefficient $C_{f}$ on the NACA 0012 airfoil is shown in Figs. 2 and 3 for the single airfoil (CASE 0) and for two airfoils in tandem (CASE 1). The agreement of both predicted parameters with experimental data is very good. Due to the incoming wake, the transition onset is moved upstream from $x / c=$ 0.62 to $x / c=0.20$ for the second airfoil. Nevertheless, the effect of the foregoing airfoil on the pressure coefficient is small.

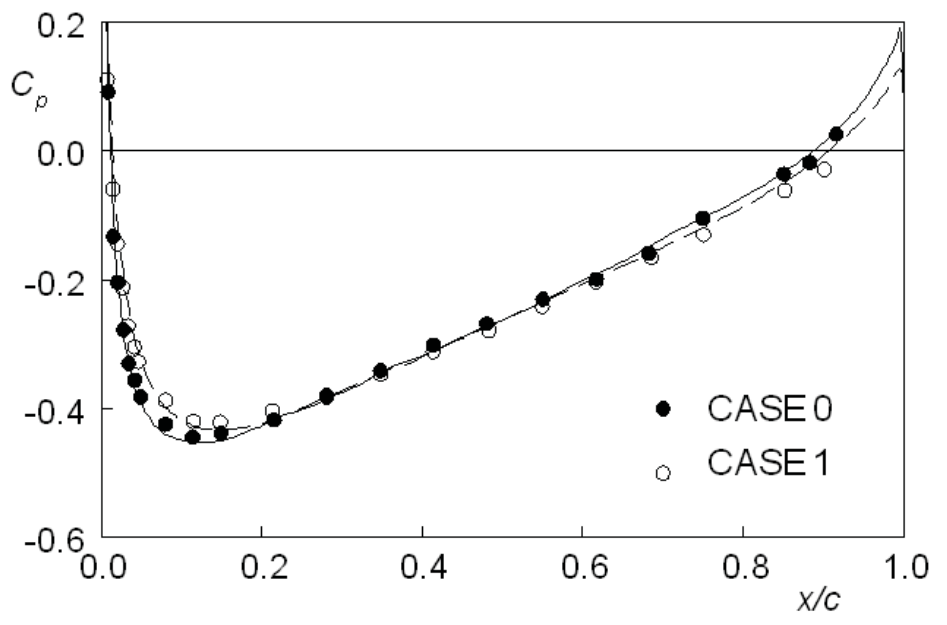

Figure 2: Distribution of the pressure coefficient $\left(\operatorname{Re}_{c}=6 \times 10^{5}, t / c=1\right)$ 


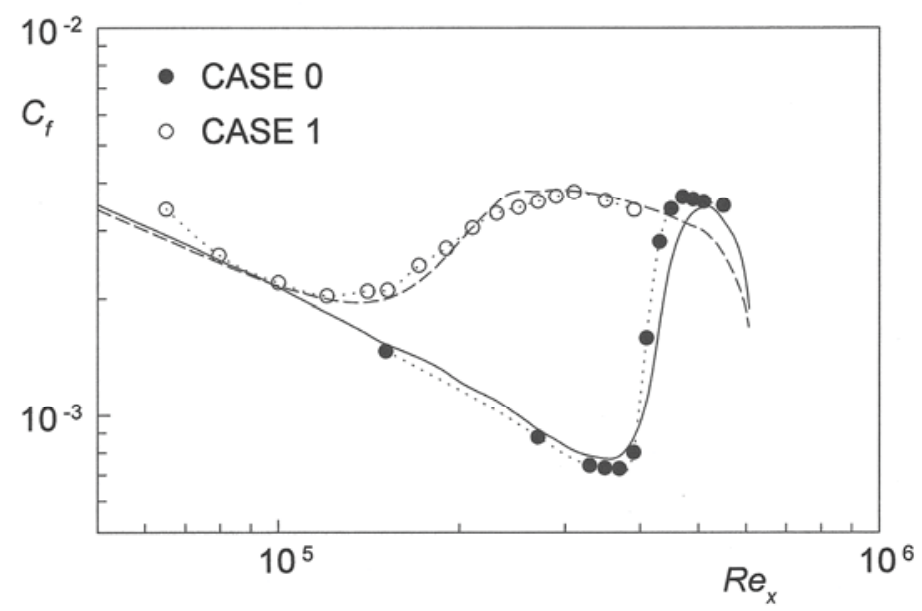

Figure 3: Distribution of the skin friction coefficient $\left(R e_{c}=6 \times 10^{5}, t / c=1\right)$

The $\gamma$-Re model of the bypass transition with the transport equation for the intermittency coefficient proposed by Langtry and Menter [7] was used for comparison. Predictions of the flow over NACA 0012 airfoils were realized by means of the SST turbulence model and the $\gamma$-Re model implemented in the commercial software ANSYS Fluent 13.0. The distribution of the skin friction coefficient is in Fig. 4 compared with experimental data for two airfoils with the distance $t / c=1$ and $R e_{c}=6 \times 10^{5}$. Due to relatively low free-stream turbulence, the transition onset in the CASE 0 is moved rather downstream and a short laminar separation bubble is predicted. On the other hand, the effect of the foregoing airfoil on the transition onset is overestimated.

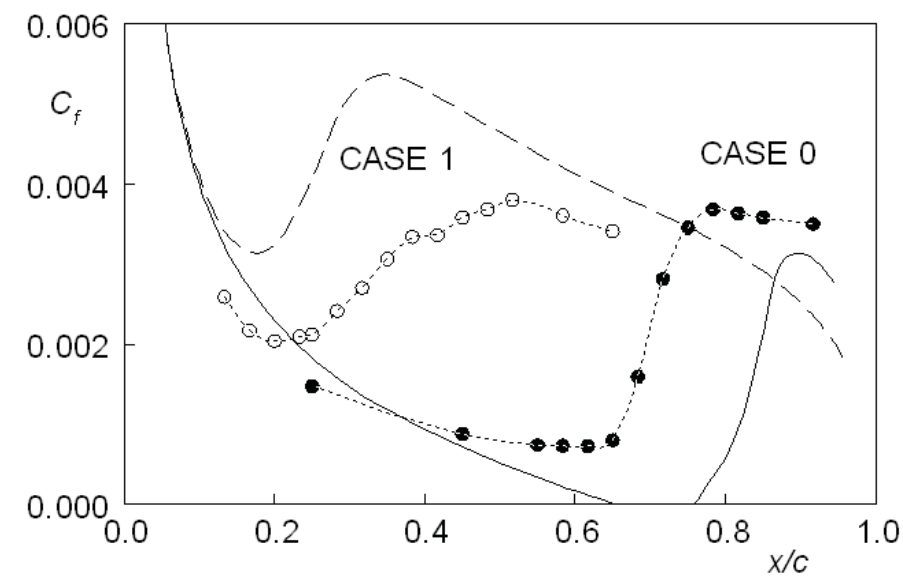

\section{Figure 4: Distribution of the skin friction coefficient obtained} by SST turbulence model and $\gamma$-Re transition model

The prediction of the flow over the airfoil is compared with the exact solution of the Navier-Stokes equations for two-dimensional incompressible laminar flow near the stagnation point of a circular cylinder with the radius $r$ corresponding to the radius of the leading edge. Using the solution of Hiemenz [17], the momentum Reynolds number and the skin friction distributions near the leading edge are given by relations

$$
\frac{R e_{\theta}}{\sqrt{R e}}=0.39375 \frac{s}{r} \quad \text { and } \quad \frac{\tau_{w}}{\rho U_{o}^{2}} \sqrt{R e}=2.4432 \frac{s}{r}
$$

where $s$ is the distance along the surface, $R e=U_{o} r / v$ is the Reynolds number based on the incoming velocity $U_{o}$ and the radius of the leading edge. This distribution of the skin 
friction near the leading edge is in Fig. 5 compared with the prediction by means of the TNT $k$ - $\omega$ model with the algebraic transition model and the SST model with the $\gamma$-Re transition model. The agreement is quite satisfactory.



Figure 5: Distribution of the skin friction near the leading edge

The dependence of the skin friction coefficient on the momentum Reynolds number $R e_{\theta}$ for the CASE 0 is shown in Fig. 6. Predicted results are compared with experimental data and empirical relations for the laminar and turbulent boundary layers on a flat plate denoted by dashed lines. Though the predicted distribution of $C_{f}$ corresponds well with experimental data (see Fig.3), the predicted momentum Reynolds numbers grows more slowly in the transition region.

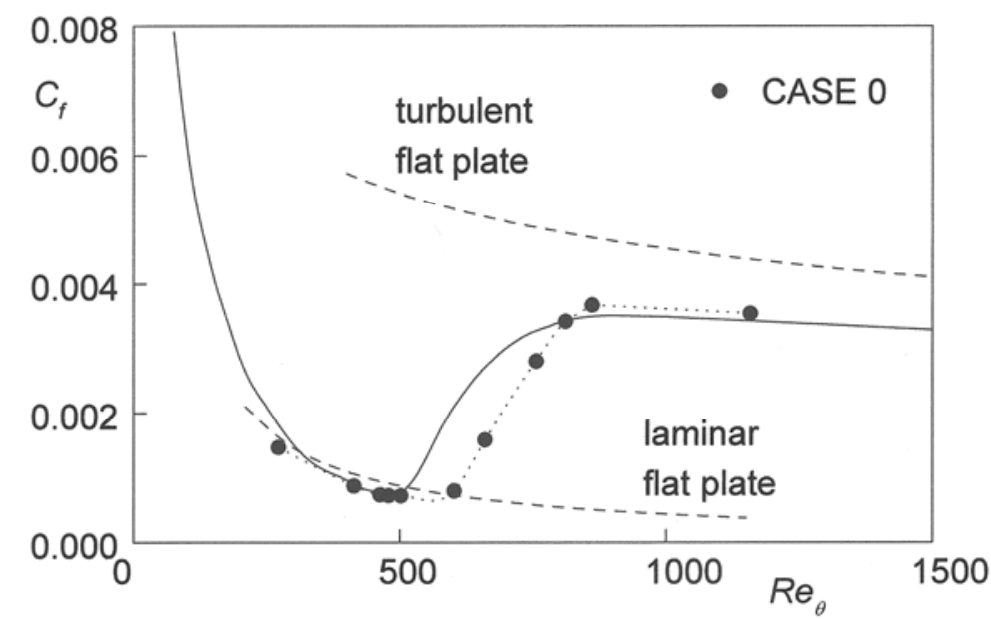

Figure 6: Dependence of the skin friction coefficient on the momentum Reynolds number

The effect of the Reynolds number $R e_{c}$ on the transition onset affected by the wake of the foregoing airfoil is shown in Fig.7. The transition onset moves upstream with the decreasing Reynolds number and the constant distance between airfoils $t / c=1$. The agreement of numerical results with experimental data is quite good except the CASE 3 $\left(R e_{c}=2 \times 10^{5}\right)$ where the shift of the transition onset is somewhat greater.

The same trends can be seen in Fig. 8 where the effect of the distance between both airfoils is shown for the constant Reynolds number $R e_{c}=2 \times 10^{5}$. The transition onset 
moves upstream with the decreasing distance between airfoils but due to the relatively low Reynolds number the agreement of predicted results with experimental data is not so good.

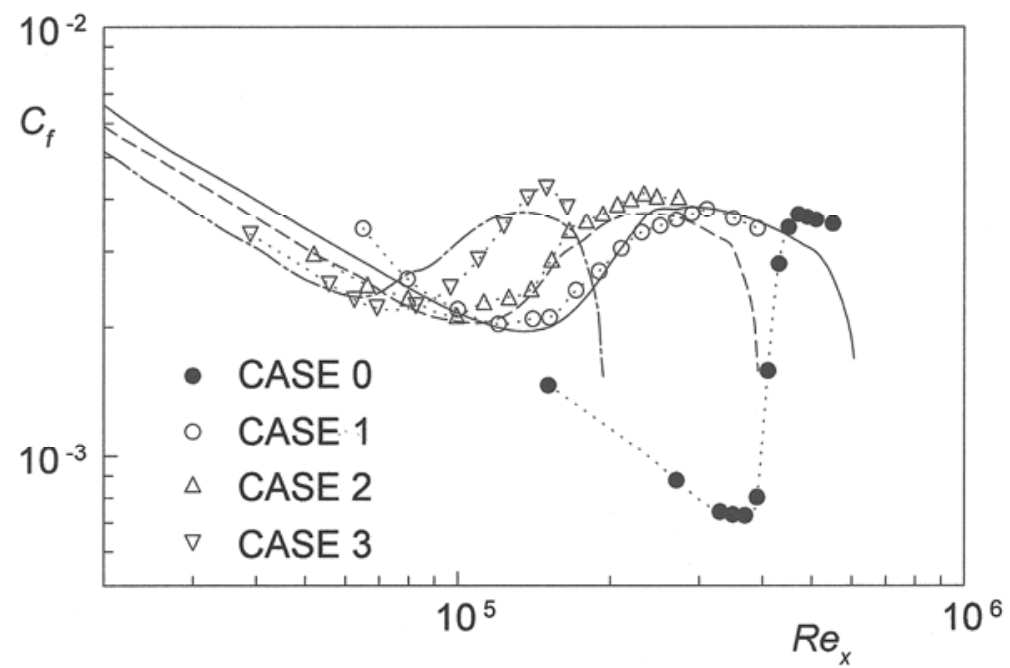

Figure 7: Effect of the Reynolds number on the transition onset

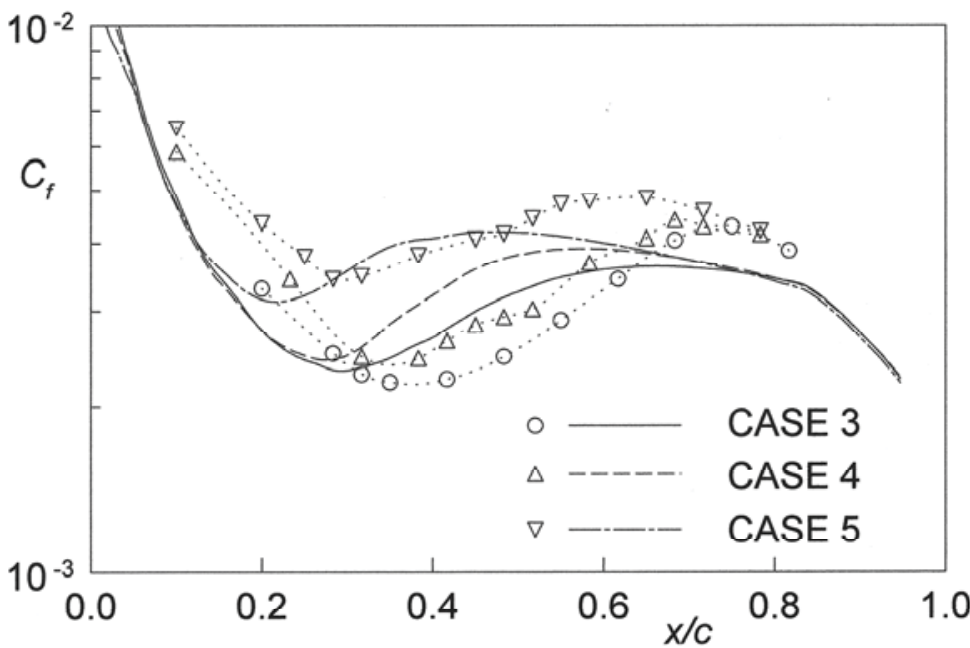

Figure 8: Effect of the distance between airfoils on the transition onset

\section{Conclusions}

The algebraic model of bypass transition taken into account different intermittency in the outer flow and in the boundary layer was applied to modelling of the transitional flow over two NACA 0012 airfoils in a tandem configuration. The ability of the transition model to predict of wake-induced transition was tested for various Reynolds number and distance between both airfoils. The agreement of predicted results with experimental data is satisfactory. Due to the modified transition criterion, the model is able to predict the laminar/turbulent transition at relatively low turbulence level. Nevertheless, a further attention should be given to estimation of relevant values of free-stream turbulence especially at lower Reynolds numbers. 


\section{ACKNOWLEDGEMENT}

The work was supported by the Research Plan AV0Z20760514 and by the grant No.101/10/1329 of the Czech Science Foundation.

\section{REFERENCES}

[1] Lee H., Kang S.-H.: Flow characteristics of transitional boundary layers on an airfoil in wakes, J. Fluids Eng., 122, 522-532, 2000

[2] Stock H.W., Haase W.: Navier-Stokes airfoil computations with $e^{N}$ transition prediction including transitional flow regimes, AIAA J., 38, 2059-2066, 2000

[3] Narasimha R.: On the distribution of intermittency in the transition region of a boundary layer, J. Aerospace Science, 24, 711-712, 1957

[4] Walters D.K., Leylek J.H.: A new model for boundary-layer transition using a single-point RANS approach, ASME, J. Turbomachinery, 126, 193-202, 2004

[5] Suzen Y.B., Huang P.G.: Modeling of flow transition using an intermittency transport equation, ASME, J. Fluids Engineering, 122, 273-284, 2000

[6] Langtry R.: A correlation-based transition model using local variables for unstructured parallelized CFD codes, PhD dissertation University Stuttgart, 2006

[7] Langtry R., Menter F.R.: Correlation-based transition modeling for unstructured parallelized computational fluid dynamics codes, AIAA J., 47, 2894-2906,2009

[8] Lodefier K., Merci B., De Langhe C., Dick E.: Intermittency based RANS bypass transition modelling, Progress in Computational Fluid Dynamics, 6, 68-78, 2006

[9] Thermann H., Niehuis R.: Unsteady Navier-Stokes simulation of a transonic flutter cascade near-stall conditions applying algebraic transition models, ASME, J. Turbomachinery, 128, 474-483, 2006

[10] Straka P., Príhoda J.: Application of the algebraic bypass-transition model for internal and external flows, In: Proc. Conf. Experimental Fluid Mechanics 2010 (EFM10), TU Liberec, 636-641, 2010

[11] Kok J.C.: Resolving the dependence on freestream values for the $k-\omega$ turbulence model, AIAA J., 38, 1292-1295, 2000

[12] Durbin P.: Near-wall turbulence closure modelling without damping functions, Theor. Comput. Fluid Dyn., 3,1, 1-13, 1991

[13] Medic G., Durbin P.A.: Toward improved prediction of heat transfer on turbine blades, ASME, J. Turbomachinery, 124, 187-192, 2002

[14] Narasimha R.: The laminar-turbulent transition zone in the boundary layer, Progress in Aerospace Science, 22, 29-80, 1985

[15] Solomon W.J., Walker G.J., Gostelow J.P.: The laminar-turbulent transition zone in the boundary layer, ASME, J. Turbomachinery, 118, 744-751, 1996

[16] Falkner V.M., Skan S.W.: Some approximate solutions of the boundary layer equations, Phil. Mag., 12, 865-896, 1930

[17] Hiemenz K.: Die Grenzschicht an einem in den gleichförmigen Flüssigkeitsstrom eingetauchten geraden Kreiszylinder, Dissertation, Göttingen, 1911 